\title{
Resolution of Metabolic Disorders and Overweight in a Patient with ACTH-independent Cushing's Syndrome after Unilateral Adrenalectomy
}

\author{
Seung Hee Yu, Yeun Sun Kim, Ki Young Lee* \\ Division of Endocrinology and Metabolism, Department of Internal Medicine, Gachon University Gil Medical Center, Incheon, Korea
}

Excessive production of cortisol by abnormal adrenocortical tissue causes clinical manifestations of Cushing's syndrome and is associated with metabolic abnormalities including abdominal obesity, hyperglycemia, dyslipidemia, and hypertension, which increase the risk for type 2 diabetes mellitus as well as vascular morbidity and mortality. Removing the cause of hypercortisolism is initially required to resolve metabolic disorders in patients with adrenal Cushing's syndrome. A 38-year-old woman with diabetes mellitus and hypertension, which were not well controlled by medications, complained of abdominal obesity, rounded face, thin limbs, and bruising. Based on clinical manifestations and laboratory findings, she was diagnosed with Cushing's syndrome due to unilateral cortisol-producing adrenal adenoma. After left adrenalectomy, the patient's blood glucose improved to a satisfactory level, and she rapidly discontinued insulin and oral glucose-lowering agent therapy. Her body mass index decreased to the normal range, and her other metabolic symptoms, dyslipidemia and hypertension, also improved significantly. She has maintained resolution of metabolic disorders and overweight for eight years since surgery without recurrence of Cushing's syndrome.

Key words: Cushing's syndrome, Metabolic diseases, Diabetes mellitus, Overweight

\author{
Received May 4, 2017 \\ Reviewed May 18, 2017 \\ Accepted May 19, 2017 \\ ${ }^{*}$ Corresponding author \\ Ki Young Lee \\ http://orcid.org/0000-0002-0333-7093 \\ Department of Internal Medicine, \\ Gachon University Gil Medical Center, \\ 24 Namdong-daero 774beon-gil, \\ Namdong-gu, Incheon 21565, Korea \\ Tel: +82-32-460-3204 \\ Fax: +82-32-460-4320 \\ E-mail: kylee@gilhospital.com
}

\section{INTRODUCTION}

Endocrine diseases concurrently present with variable clinical manifestations and metabolic abnormalities. ${ }^{1}$ It is important to identify the cause of disease and treat the disease using an appropriate approach. Cushing's syndrome, characterized by the secretion of excess cortisol by the adrenal gland or ectopic sites, is one of the leading causes of metabolic disorders due to hypercortisolism and frequently induces diabetes mellitus, dyslipidemia, hypertension, and obesity. The clinical courses of these metabolic disorders, which are associated with Cushing's syndrome, are often more severe than in cases without hypercortisolism and cannot be controlled without addressing the primary cause of disease. ${ }^{2}$
We describe a patient with severe unregulated endocrine metabolic diseases, including diabetes mellitus, dyslipidemia, hypertension, and overweight, whose condition improved dramatically after surgical treatment for Cushing's syndrome and include a review of the literature.

\section{CASE REPORT}

Written informed consent was obtained from the patient. A 38-year-old woman visited the endocrine department of our hospital with complaints of weight gain, presenting with increased abdominal obesity, rounded face, thin limbs, and easy bruising that had persisted for 5 years. She had been diagnosed with diabetes 
mellitus 4 months prior to presentation and had been taking amlodipine $5 \mathrm{mg}$ twice daily to control high blood pressure for 3 years. The patient was suspected to have Cushing's syndrome based on these manifestations and past history. The results of screening tests were positive: serum cortisol level $22.9 \mu \mathrm{g} / \mathrm{dL}$ (reference range: 9.4$26.0 \mu \mathrm{g} / \mathrm{dL}$ ) at 8:00 am and 24-hour urinary free cortisol level 584.0 $\mu \mathrm{g} /$ day (reference range: 55.5-286.0 $\mu \mathrm{g} / \mathrm{day}$ ). The patient was admitted to our hospital for further evaluation of Cushing's syndrome.

On admission, she had a high blood pressure of $170 / 80 \mathrm{mmHg}$ despite amlodipine $5 \mathrm{mg}$ twice daily for hypertension. Her height was $160 \mathrm{~cm}$ and body weight was $62 \mathrm{~kg}$, with a body mass index (BMI) of $24.2 \mathrm{~kg} / \mathrm{m}^{2}$ and was overweight according to Asia-Pacific WHO criteria for adults. ${ }^{3}$ Her waist circumference was $73 \mathrm{~cm}$. Her total cholesterol, triglycerides (TG), high-density lipoprotein cholesterol (HDL-cholesterol), and low-density lipoprotein cholesterol (LDL-cholesterol) were $323 \mathrm{mg} / \mathrm{dL}, 349 \mathrm{mg} / \mathrm{dL}, 59 \mathrm{mg} / \mathrm{dL}$, and $203 \mathrm{mg} / \mathrm{dL}$, respectively. Her fasting glucose level was $183 \mathrm{mg} / \mathrm{dL}$, fasting insulin level was $4.81 \mu \mathrm{IU} / \mathrm{mL}$, and homeostasis model assessment of insulin resistance was 2.17 . Glycated hemoglobin ( $\mathrm{HbAlc}$ ) was 8.9\%, and the patient was receiving treatment constituting 14 units of insulin detemir plus $1,000 \mathrm{mg}$ of metformin for blood glucose control.

In the 48-hour low-dose dexamethasone suppression test, her serum cortisol level was $24.6 \mu \mathrm{g} / \mathrm{dL}$ and was not suppressed to less than $2 \mu \mathrm{g} / \mathrm{dL}$. Plasma adrenocorticotropic hormone (ACTH) concentration was $1.59 \mathrm{pg} / \mathrm{mL}$ (reference range: $10-60 \mathrm{pg} / \mathrm{mL}$ ) and was lower than the normal range. Abdominopelvic computed tomography was performed to identify the cause of ACTH-independent Cushing's syndrome and revealed a $3.8 \mathrm{~cm}$ homogenous enhancing mass in the left adrenal gland (Fig. 1). Levels of other hormones including 24-hour urine metanephrine, epinephrine, norepinephrine, vanillylmandelic acid, 17-hydroxycorticosteroid and plasma aldosterone concentration and plasma renin activity were within normal ranges. Based on these findings, the patient was diagnosed with ACTH-independent Cushing's syndrome due to a left adrenal adenoma.

Laparoscopic left adrenal gland removal was performed 2 weeks after diagnosis of Cushing's syndrome. The pathologic finding was cortical adenoma in the left adrenal gland. After left adrenalectomy, the patient's metabolic abnormalities improved, especially those in-

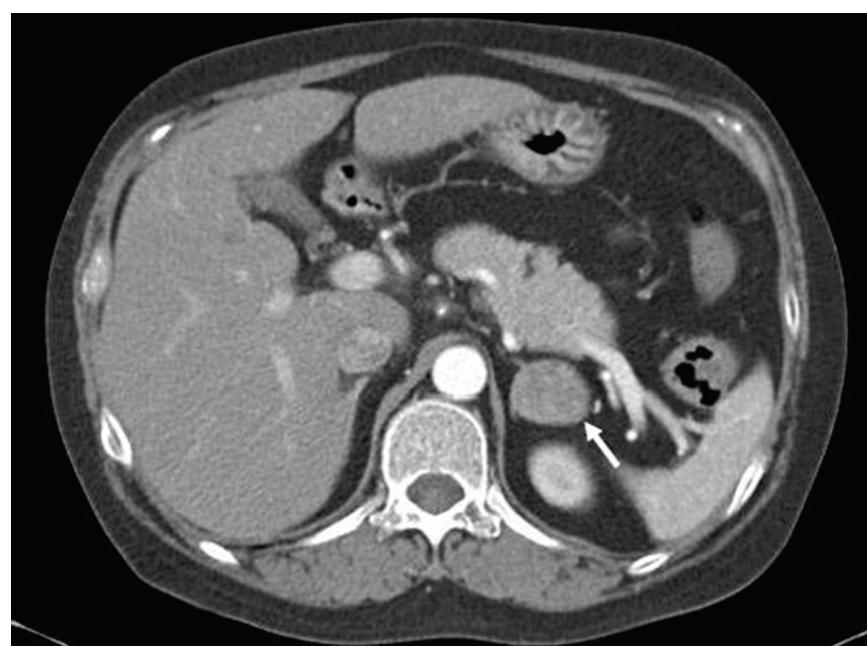

Figure 1. Abdominopelvic computed tomography showed a $3.8 \mathrm{~cm}$ homogenous enhancing mass in the left adrenal gland (arrow).

dicating glucose and lipid metabolism (Table 1). Her blood glucose level rapidly decreased compared to preoperative blood glucose level, and we stopped her insulin therapy 2 weeks after surgery. Over 3 months, the patient's HbAlc level improved from 8.9\% to $7.0 \%$ despite the use of prednisolone $7.5 \mathrm{mg}$ daily as steroid replacement therapy. Fifteen months after surgery, the patient stopped all oral glucose-lowering agents. Thereafter, oral steroid agent therapy was successfully discontinued, and plasma ACTH recovered to the normal range. At that time, the patient's body weight was $52 \mathrm{~kg}$, or about $10 \mathrm{~kg}$ less than before left adrenalectomy. Her BMI was $20.3 \mathrm{~kg} / \mathrm{m}^{2}$ or within the normal range. Her waist circumference was $68 \mathrm{~cm}$. Lipid profile, including total cholesterol, TG, HDL-cholesterol, and LDL-cholesterol, also improved. Eight years after surgery, the patient is currently taking only amlodipine $5 \mathrm{mg}$ once daily for hypertension treatment, and her blood pressure remains stable.

\section{DISCUSSION}

Benign adrenocortical tumors are the most common cause of ACTH-independent Cushing's syndrome. ${ }^{1,4}$ In some studies of obese patients with type 2 diabetes, especially those with poor blood glucose control and hypertension, the reported prevalence of Cushing's syndrome is between $2 \%$ and $5 \%$. These data suggest that Cushing's syndrome is more common than previously believed. ${ }^{4}$ Excessive production of cortisol by abnormal adrenocorti- 
Table 1. Laboratory improvement in a patient with ACTH-independent adrenal adenoma after surgery

\begin{tabular}{|c|c|c|c|c|c|}
\hline & Before unilateral adrenalectomy & & After uni & alectomy & \\
\hline & July 2009 & October 2010 & July 2012 & June 2015 & December 2016 \\
\hline Body weight (kg) & 62 & 52 & - & - & - \\
\hline $\mathrm{BMI}\left(\mathrm{kg} / \mathrm{m}^{2}\right)$ & 24.2 & 20.3 & - & - & - \\
\hline Cortisol ( $\mu \mathrm{g} / \mathrm{dL})$ & 22.9 & 6.6 & 13.5 & 18.4 & 15.7 \\
\hline ACTH $(p g / m L)$ & 1.59 & 34.5 & 70.32 & 85.5 & 52.8 \\
\hline Fasting glucose (mg/dL) & 183 & 96 & 90 & 87 & 94 \\
\hline $\mathrm{HbA} 1 \mathrm{c}(\%)$ & 8.9 & 6.1 & 5.1 & 5.2 & 5.4 \\
\hline Total cholesterol (mg/dL) & 323 & 177 & 209 & - & - \\
\hline Triglycerides (mg/dL) & 349 & 120 & 139 & - & - \\
\hline HDL-cholesterol (mg/dL) & 59 & 44 & 68 & - & - \\
\hline LDL-cholesterol (mg/dL) & 203 & 109 & 113 & - & - \\
\hline
\end{tabular}

ACTH, adrenocorticotropic hormone; BMI, body mass index; HbA1C, glycated hemoglobin; HDL-cholesterol, high-density lipoprotein cholesterol; LDL-cholesterol, low-density lipoprotein cholesterol.

cal tissue causes clinical manifestations of Cushing's syndrome and is associated with metabolic abnormalities including abdominal obesity, hyperglycemia, dyslipidemia, and hypertension, all of which increase the risk for type 2 diabetes mellitus as well as vascular morbidity and mortality. The most common of the metabolic abnormalities is weight gain, which is usually central but can be general in distribution, often accompanied by thickening of the facial fat, which rounds the face. ${ }^{5}$ Cortisol is an important hormone in the regulation of glucose metabolism, and endogenous hypercortisolism is involved in not only abdominal obesity, but also development of hyperglycemia, which induces diabetes mellitus in Cushing's syndrome. Jeong et al. ${ }^{6}$ reported that insulin resistance was significantly increased and insulin secretion was significantly decreased by exacerbation of hypercortisolism in patients with Cushing's syndrome. Dyslipidemia, characterized by increased very low-density lipoprotein, LDL-cholesterol, TG, and total cholesterol levels with decreased HDL-cholesterol and hepatic steatosis, have also been reported in patients with Cushing's syndrome. ${ }^{7}$ Excess cortisol predisposes individuals to hypertension, as they lack nocturnal blood pressure dipping and experience increased cardiovascular risk. ${ }^{8}$

In this case, the patient had variable metabolic abnormalities including sudden onset of weight gain, diabetes mellitus, increased total cholesterol, TG and LDL-cholesterol, and poorly controlled blood pressure despite medication, caused by ACTH-independent Cushing's syndrome due to left adrenal adenoma.

It is necessary to remove the cause of hypercortisolism to resolve metabolic abnormalities, and the treatment of choice in patients with adrenal Cushing's syndrome is surgical adrenal resection for unilateral adrenal adenomas. However, insulin resistance, impairment of insulin secretion, and increased blood pressure can persist even after correction of cortisol excess and diabetes, leading to a need for long-term hypertension treatment. ${ }^{9}$ Accumulating evidence suggests that cured Cushing's syndrome patients have increased morbidity and mortality despite endocrine control. ${ }^{2}$

The most interesting points of our case were that the patient showed dramatic improvement regarding metabolic abnormalities within a short period of time after surgery to remove a cortisol-secreting left adrenal adenoma and has remained in a state of metabolic resolution without recurrent Cushing's syndrome or metabolic disorders for a long period. Two weeks after surgery, she no longer needed insulin therapy despite receiving steroid replacement therapy and eventually achieved biochemical resolution of diabetes mellitus without receiving any oral glucose-lowering medication.

Treatment of Cushing's syndrome should aim to cure hypercortisolism and to eliminate any tumors that threaten the patient's health, while minimizing the chance of endocrine deficiency or long-term dependence on medications. ${ }^{1}$ The outcome of our case suggests that it is important to recognize whether there are any other diseases requiring rapid treatment in patients with uncontrolled or severe endocrine disorders. Our patient experienced significant improvements of diabetes mellitus, hypertension, overweight, and dyslipidemia after treatment of adrenal Cushing's syndrome. At the time of writing the patient has maintained a state of normal glucose tolerance for 8 years. 


\section{CONFLICTS OF INTEREST}

The authors declare no conflict of interest.

\section{REFERENCES}

1. Orth DN. Cushing's syndrome. N Engl J Med 1995;332:791803.

2. Resmini E. Persistent comorbidities in cushing's syndrome after endocrine cure. Adv Endocrinol 2014;2014:231432.

3. Bassett J; International Diabetes Institute; World Health Organization Regional Office for the Western Pacific; International Association for the Study of Obesity; International Obesity Task Force. The Asia-Pacific perspective: redefining obesity and its treatment. Melbourne: Health Communications Australia; 2000. p. 15-21.

4. Newell-Price J, Bertagna X, Grossman AB, Nieman LK. Cushing's syndrome. Lancet 2006;367:1605-17.
5. Ross EJ, Linch DC. Cushing's syndrome--killing disease: discriminatory value of signs and symptoms aiding early diagnosis. Lancet 1982;2:646-9.

6. Jeong IK, Kim SH, Chung JH, Min YK, Lee MS, Lee MK, et al. The change of insulin sensitivity and insulin secretion according to glucose metabolism status in patients with Cushing's syndrome. J Korean Soc Endocrinol 2003;18:392-403.

7. Arnaldi G, Scandali VM, Trementino L, Cardinaletti M, Appolloni G, Boscaro M. Pathophysiology of dyslipidemia in Cushing's syndrome. Neuroendocrinology 2010;92 Suppl 1: 86-90.

8. Mancini T, Kola B, Mantero F, Boscaro M, Arnaldi G. High cardiovascular risk in patients with Cushing's syndrome according to 1999 WHO/ISH guidelines. Clin Endocrinol (Oxf) 2004;61:768-77.

9. Mazziotti G, Formenti AM, Frara S, Maffezzoni F, Doga M, Giustina A. Diabetes in Cushing disease. Curr Diab Rep 2017; $17: 32$. 